\title{
Patisiran, an RNAi therapeutic for the treatment of hereditary transthyretin-mediated amyloidosis
}

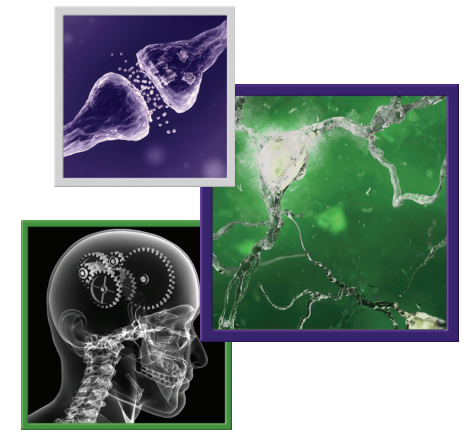

\author{
Arnt V Kristen*,1,2, Senda Ajroud-Driss ${ }^{3}$, Isabel Conceição ${ }^{4}$, Peter Gorevic ${ }^{5}$, Theodoros \\ Kyriakides $^{6}$ \& Laura Obici $^{7}$ \\ ${ }^{1}$ Department of Cardiology, University of Heidelberg, Heidelberg, D-69120, Germany \\ ${ }^{2}$ Cardiovascular Center Darmstadt, Darmstadt, 64287, Germany \\ ${ }^{3}$ Department of Neurology, Northwestern University, Feinberg School of Medicine, Chicago, IL 60611, USA \\ ${ }^{4}$ Centro Hospitalar Lisboa Norte, Hospital de Santa Maria, Universidade de Lisboa, Faculdade de Medicina, 1649-028, Portugal \\ ${ }^{5}$ Department of Medicine, Mount Sinai Medical Center, New York, NY 10029, USA \\ ${ }^{6}$ The Cyprus Institute of Neurology \& Genetics, Nicosia, 1683, Cyprus \\ ${ }^{7}$ Amyloidosis Research \& Treatment Centre, Fondazione IRCCS Policlinico San Matteo, Pavia, 27100, Italy \\ *Author for correspondence: Tel.: +49 6151 297620; Fax: +49 6151 176117; kristen@kardio-darmstadt.de
}

\section{Practice points}

Mechanism of action

- Patisiran is a double-stranded small interfering RNA that targets a sequence within the transthyretin (TTR) messenger RNA that is conserved across wild-type and all TTR variants to decrease hepatic production of mutant and wild-type TTR.

Pharmacology

- Patisiran has been consistently shown to achieve rapid onset (peak effect within $\sim 2$ weeks), robust ( $\sim 80-90 \%)$ and sustained ( $>36$ months) reduction of serum TTR when dosed at $0.3 \mathrm{mg} / \mathrm{kg}$ body weight every 3 weeks.

- Prior treatment with TTR stabilizers did not interfere with the pharmacological activity of patisiran.

Clinical efficacy

- In the Phase III APOLLO study, patisiran treatment led to statistically significant improvements in polyneuropathy (modified Neuropathy Impairment Score +7 ) and Norfolk Quality of Life-Diabetic Neuropathy questionnaire measures compared with placebo in patients with hereditary TTR-mediated amyloidosis.

- The beneficial effects of patisiran treatment on polyneuropathy and quality of life were consistent across all major subgroups (age, gender, race, region, genotype, neuropathy impairment score at the start of treatment, familial amyloid polyneuropathy stage, previous TTR stabilizer use and cardiac involvement) in the APOLLO study.

- Patisiran improved multiple clinical end points compared with placebo in the APOLLO study, including motor strength, disability, gait speed, nutritional status and autonomic symptoms.

- Patisiran treatment was associated with improvement in cardiac structure and function in a predefined cardiac subpopulation in the APOLLO study, with significant reductions in left ventricular wall thickness, left ventricular longitudinal strain and $\mathrm{N}$-terminal prohormone of brain-type natriuretic peptide levels at 18 months.

Safety

- Patisiran was generally well tolerated and showed a consistent safety profile in patients with hereditary TTR-mediated amyloidosis across all phases of clinical development, in which 218 patients were exposed for periods of up to 4 years.

Hereditary transthyretin-mediated amyloidosis is a rapidly progressive, heterogeneous disease caused by the accumulation of misfolded transthyretin protein as amyloid fibrils at multiple sites, and is characterized by peripheral sensorimotor neuropathy, autonomic neuropathy and/or cardiomyopathy. Current treatment options have limited efficacy and often do not prevent disease progression. Patisiran is a novel RNA interference therapeutic that specifically reduces production of both wild-type and mutant transthyretin protein. In Phase II, III and long-term extension studies in patients with hereditary transthyretin-mediated amyloidosis, patisiran has consistently slowed or improved progression of neuropathy. In addition, the Phase III trial demonstrated significant improvements in quality of life measures and indicators of cardiomyopathy. Here, we highlight efficacy and safety data from the patisiran clinical trial programme. 
First draft submitted: 30 August 2018; Accepted for publication: 25 October 2018; Published online: 27 November 2018

Keywords: cardiomyopathy • clinical trials • disease-modifying therapy • hATTR amyloidosis • patisiran - polyneuropathy

\section{Introduction}

Amyloidosis

The systemic amyloidoses are a family of heterogeneous conditions caused by the deposition of misfolded proteins as insoluble amyloid fibrils in multiple organs, which ultimately causes disruption of normal tissue structure and function [1,2]. Up to 30 different precursor proteins capable of amyloid fibril formation have been identified [3], which lead to disorders that have a diverse clinical presentation affecting the kidneys, liver, heart and peripheral nervous system [2]. The clinical course and prognosis are determined by the specific precursor protein, and the organ systems involved. The more common forms of systemic acquired amyloidosis are amyloid A (AA) amyloidosis and light chain (AL) amyloidosis, involving serum AA protein and immunoglobulin light chain proteins, respectively. AA amyloidosis mainly affects the kidney, and nervous system involvement is rare [4], whereas peripheral neuropathy occurs in approximately $26 \%$ of patients with AL amyloidosis [5]. Transthyretin-mediated (ATTR) amyloidosis results from misfolding of the precursor protein transthyretin (TTR), which is due either to a TTR mutation leading to the hereditary form of the disease (hATTR amyloidosis), or to deposition of wild-type (wt) TTR protein leading to an acquired form of the disease [6], previously known as senile systemic amyloidosis [7].

\section{hATTR amyloidosis}

hATTR amyloidosis is a rare, autosomal dominantly inherited, progressively debilitating and life-threatening disease caused by mutations in the TTR gene. The resulting misfolded TTR proteins accumulate as amyloid deposits at multiple sites including nerves, heart and GI tract [8-11] culminating in intractable peripheral sensorimotor neuropathy and in many cases autonomic neuropathy and/or cardiomyopathy [2,9,10,12]. Although the exact prevalence is unknown, hATTR amyloidosis has been reported in more than 30 countries [13] with a current estimated worldwide prevalence of 50,000 individuals [10]. However, in certain areas of Portugal, Sweden or Japan, where hATTR amyloidosis is endemic, disease prevalence can reach up to 1 in 1000 [10,14,15].

Pathophysiology

TTR is a homotetrameric plasma protein, comprising four identical 127 -amino acid $\beta$-sheet monomers bound together as a pair of dimers. It is produced predominantly by the liver [9,16] but also synthesized by the retinal pigment epithelium and choroid plexus [17]. TTR functions primarily as a transport protein for vitamin A and for approximately $15 \%$ of circulating plasma thyroxine [17-19]. However, in patients with hATTR amyloidosis the tetramer dissociates, with the rate-limiting step to amyloid fibril formation being the initial dissociation of the TTR tetramer into two dimers (Figure 1). The dimers further dissociate into monomers which undergo conformational transformation to highly ordered and abnormal amyloid fibril aggregates $[9,10]$. TTR mutations favour proteolytic remodelling and destabilization of the tetramer protein leading to increased formation of misfolded amyloidogenic $\mathrm{C}$-terminal fragments and full-length monomers which can more readily form aggregates $[9,10,20,21]$. As most patients with hATTR amyloidosis are heterozygous for the TTR mutations, amyloid deposits often contain a mixture of mutated and wt TTR [22].

\section{Clinical manifestations \& disease characteristics}

Deposition of amyloid across multiple systems in patients with hATTR amyloidosis results in a heterogeneous clinical presentation that includes sensorimotor and/or autonomic neuropathy, cardiac symptoms and less commonly vitreous amyloid or nephropathy, with many patients presenting with concurrent, variable symptoms [2,9]. Sensory neuropathy manifests as pain, numbness and impaired thermal sensitivity, which generally begins distal at the lower limbs and progresses proximally reaching the upper limbs at later stages [6,23]. Motor involvement in the distal lower limbs leads to muscle weakness and increasing difficulty with walking, with loss of balance and impaired gait [24]. Autonomic dysfunction includes sweating abnormalities, sexual impotence, unintentional weight loss, disturbances of GI motility (diarrhea, constipation, nausea and vomiting), urinary retention and orthostatic hypotension [2,6]. Amyloid infiltrates many cardiovascular structures, leading to a wide range of symptoms including cardiomyopathy, arrhythmia, dyspnea, shortness of breath and edema [25]. Of note, carpal tunnel syndrome can often present at the 


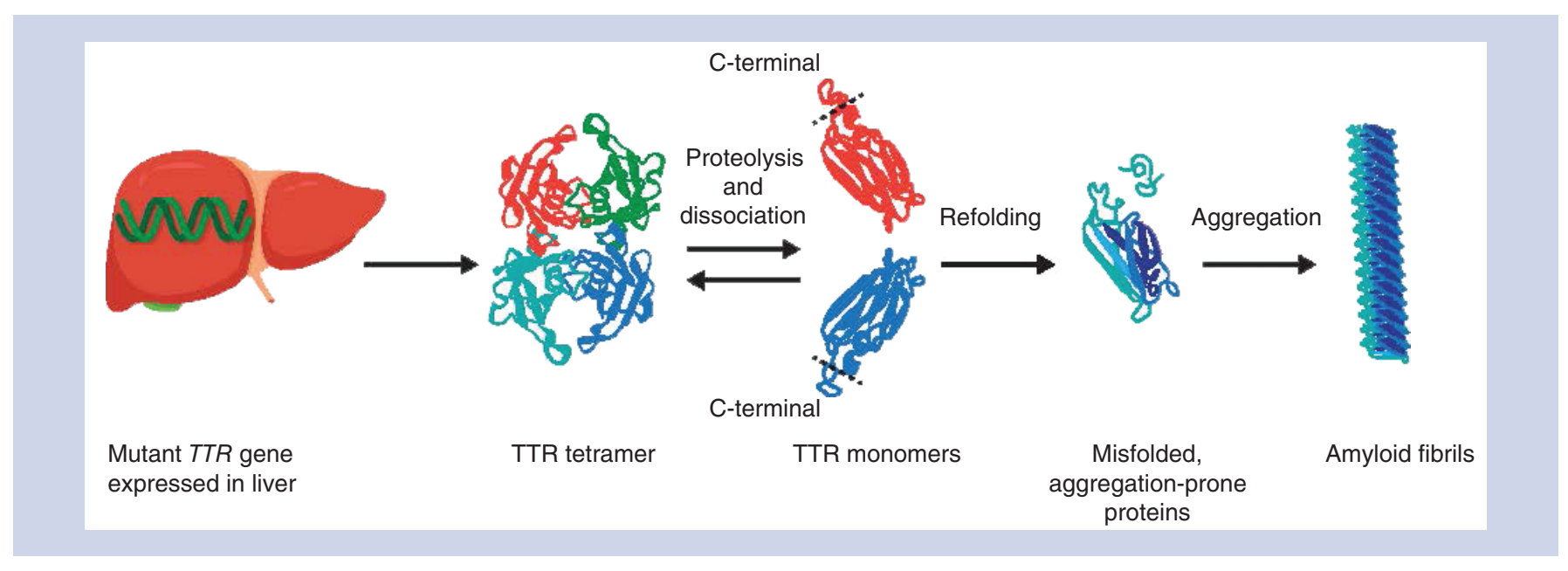

Figure 1. Schematic diagram of the pathophysiology of transthyretin-mediated amyloidosis. In hereditary transthyretin-associated (ATTR) amyloidosis, a mutated form of TTR protein is produced by the liver. TTR mutations favor proteolytic remodeling and destabilization of the tetramer protein leading to increased formation of misfolded amyloidogenic proteins, including full-length monomers and C-terminal fragments, which rapidly aggregate into abundant amyloid fibrils. The aggregation-prone C-terminal is produced from enzymatic digestion of the TTR protein, and is found across patients with different TTR mutations, and also in patients with WT ATTR amyloidosis. The extracellular amyloid deposits also contain nonfibrillar components such as calcium, serum amyloid P component and sulphonated glycosaminoglycans.

TTR: Transthyretin; wt: Wild-type.

early stages of disease in patients with predominant cardiomyopathy, but may not be recognized as a symptom of hATTR amyloidosis [26]. Other symptoms associated with hATTR amyloidosis include renal impairment [6] and ocular involvement such as vitreous opacities, glaucoma and pupillary disorders [6].

To date, over 130 TTR mutations have been reported [27], although not all appear to be amyloidogenic [28]. Investigation of different TTR mutations indicates that they may vary in their clinical manifestations, geographical distribution and relationship to patient outcome [27,29]. Historically, hATTR amyloidosis was described by its predominant clinical manifestation, either familial amyloidotic polyneuropathy (FAP) or familial amyloidotic cardiomyopathy. The most common TTR mutation is Val30Met (more recently described as Val50Met), which was first identified in an endemic region of Portugal [30] and is predominantly associated with an early-onset neuropathic phenotype [13]. By comparison, Val122Ile, frequently found in African-Americans, is associated with cardiomyopathy [31,32]. Although genotype-phenotype correlations have been reported, numerous disease manifestations can develop concurrently and phenotypic variability among patients with the same genotype is often observed. A mixed phenotype is thus common across many mutations [33], so hATTR amyloidosis has been described as a multisystem disease [9,10,12,29,34-36].

The age of symptom onset is typically between the second and ninth decades, and substantial variation is observed across different populations [6]. Within endemic regions of Portugal and Japan, patients presenting before the age of 50 years are described as early onset, typically with predominant symptoms of polyneuropathy and severe autonomic dysfunction [37]. In nonendemic countries and endemic regions of Sweden, patients usually present from 50 years of age with a broader spectrum of clinical manifestations, and often milder autonomic involvement [16]. Patients with WT or hATTR amyloidosis and cardiomyopathy typically present with late-onset disease in their 60s [6].

The heterogeneity in presenting symptoms, disease penetrance and age of onset often leads to misdiagnosis of hATTR amyloidosis [34,36]. hATTR amyloidosis that presents predominantly with polyneuropathy is often misdiagnosed as chronic inflammatory demyelinating polyneuropathy, which also involves weakness, sensory symptoms and motor symptoms [38,39]. hATTR amyloidosis with cardiomyopathy tends to occur in older patients, and is often misdiagnosed as hypertrophic cardiomyopathy or arterial hypertension, due to the overlap in signs and symptoms and the increased prevalence of these diseases in the elderly population [10].

Early diagnosis and initiation of treatment are critical as rapid progression of neurological and cardiac symptoms often occurs in patients with hATTR amyloidosis $[8,40]$. The disease usually follows an aggressive unrelenting 
course beginning with neuropathy and often GI disturbances and autonomic dysfunction in the absence of impaired ambulation (FAP stage 1/polyneuropathy disability [PND] score 1). With increasing motor weakness, mobility declines and requirement for assistance with ambulation increases (FAP stage 2/PND score II/IIIa/IIIb). FAP stage 3 (PND score IV) is characterized by worsening of neuropathy, cachexia, diarrhoea/constipation and dizziness/fainting, with the patient becoming wheelchair-bound [6,41-43]. Patients with cardiac symptoms experience a similar progressive decline in cardiac and physical function [44,45].

The progressive increase in symptom severity and disability leads to deteriorating quality of life (QoL) and loss of physical function. Death often results from cachexia, cardiac insufficiency or infection [43,46]. The overall median survival in patients with hATTR amyloidosis is 4.7 years following diagnosis, and 3.4 years in patients presenting predominantly with cardiomyopathy [47-49]. However, prognosis can vary according to patient characteristics [43]. For example, late-onset disease typically has a worse prognosis than early-onset disease; median survival of 7 and 11 years from disease onset has been reported for patients with late-onset [42] and early-onset Val30Met disease, respectively [41].

Preventing or reversing the deterioration in symptoms and QoL thus represents a key objective in the management of patients with hATTR amyloidosis. Over the last 20 years, there has been marked progress in the development of novel treatment approaches for hATTR amyloidosis. However, currently available or approved treatment options are limited and only recommended for specific patient populations, thus highlighting a clear unmet treatment need. Optimal clinical management of hATTR amyloidosis is also challenging as the heterogeneous nature of this disease necessitates a cross-disciplinary approach, involving neurology, gastroenterology and cardiology specialties.

\section{Current therapies for hATTR amyloidosis}

Symptomatic relief

In patients with hATTR amyloidosis the relief of symptoms is key to improving QoL. Indeed for some patient populations, such as those with cardiac involvement, it remains the only available treatment strategy. The main categories of symptomatic therapy available are neuropathic pain medications (e.g., gabapentin, pregabalin and duloxetine), motility drugs which address GI symptoms (metoclopramide, polycarbophil calcium and loperamide) and medications and devices for the treatment and management of cardiac symptoms such as heart failure, atrial fibrillation and orthostatic hypotension (loop diuretics, angiotensin-converting enzyme inhibitors, pacemaker and $\beta$-blockers) $[6,10,31,46]$. However, robust data on the benefit of angiotensin-converting enzyme inhibitors, angiotensin blockers or $\beta$-blockers in ATTR amyloidosis with cardiomyopathy are currently lacking [50]. Furthermore, some drugs may also exacerbate the symptoms of this disease [10,46]. For example, loop diuretics may lead to reduced cardiac output and hypotension if used at high doses, while certain calcium channel blockers and digoxin may bind to amyloid fibrils promoting hyperphysiological effects and causing rhythm disturbances, respectively $[10,46]$.

Orthotopic liver transplantation

As TTR is produced predominantly in the liver, orthotopic liver transplantation (OLT) was introduced in the 1990 s as a rational therapy for hATTR amyloidosis by preventing the production of mutant TTR [6,51]. OLT is a well-recognized and effective treatment in some patient subsets, with long-term observations of patients receiving OLT showing regression of amyloid deposits and an overall patient survival rate at 5 years of $77 \%$ [52]. However, survival post-OLT is influenced by specific characteristics including modified body mass index, disease onset, disease duration prior to OLT and mutations other than Val30Met [6,51,53]. Indeed, the benefits of OLT have largely been restricted to early-stage Val30Met patients with polyneuropathy but without cardiac involvement [6,51].

With respect to QoL, results of a Dutch study of nine patients who had undergone OLT suggested that improvements in QoL are limited; domains relating to physical well-being were significantly lower compared with non-hATTR amyloidosis liver transplant patients, and continued to deteriorate [54]. In addition, there is evidence that progression of neuropathy and/or cardiomyopathy may continue in some patients following OLT due to deposition of WT TTR $[46,55,56]$. In a multi-national study of 362 patients with hATTR amyloidosis, $~ 33 \%$ of patients progressed within 3-5 years (PND score worsening) post-OLT [55]. In addition, OLT does not affect variant TTR production in the eye or brain, and therefore CNS and ocular manifestations are not affected $[57,58]$.

As with any transplantation procedure, OLT is limited by organ availability and carries the risks associated with surgery including hepatic artery thrombosis, infection, drug toxicities and de novo malignancies associated with long-term immunosuppression [10,59]. 
TTR tetramer stabilizers

An alternative treatment strategy for hATTR amyloidosis is to increase the stability of circulating TTR tetramers, hence preventing their dissociation to monomers and subsequent amyloid fibril formation. Both tafamidis and diflunisal have been shown to bind selectively to TTR at the thyroxine-binding site $[31,60]$ and to inhibit misfolding of the TTR protein $[6,10]$.

\section{Tafamidis}

Tafamidis (Vyndaqe ${ }^{\circledR}$ ) is an oral, small molecule TTR stabilizer [60] approved in Europe and selected countries in Asia and Latin America (Mexico, Brazil, Argentina, Japan and Israel), with the EMA indicating Vyndaqel ${ }^{\circledR}$ for the treatment of hATTR amyloidosis in adult patients with stage 1 symptomatic polyneuropathy to delay peripheral neurological impairment [61]. In a pivotal, placebo-controlled Phase II/III trial, tafamidis treatment was assessed in patients with TTR Val30Met mutation and FAP stage 1 neuropathy [62]. The coprimary end points of this study were the Neuropathy Impairment Score (NIS)-Lower Limbs (NIS-LL) responder analysis (<2-point worsening) and change from baseline in Norfolk Quality of Life-Diabetic Neuropathy (Norfolk QoL-DN) total score (TQOL). In the intent-to-treat (ITT) primary analysis population at 18 months there was a nonsignificant trend towards more responders in the tafamidis group than in the placebo group (45.3 vs $29.5 \% ; \mathrm{p}=0.068$ ) and the change from baseline in TQOL score between the treatment groups was nonsignificant. In patients with evaluable data at 18 months (efficacy evaluable population), significantly more tafamidis-treated patients achieved a treatment response (60 vs 38.1\%; $\mathrm{p}=0.041)$ and tafamidis-treated patients demonstrated better preservation of TQOL (0.1 vs 8.9 points; $\mathrm{p}=0.045$ ) [62]. Tafamidis was generally well tolerated, with a similar incidence of adverse events (AEs) in the two treatment arms. Although tafamidis demonstrated some efficacy in this study, its effect on slowing disease progression was diminished in a study of patients with late-onset Val30Met hATTR amyloidosis and more advanced disease [63]. At baseline, patients had NIS-LL $>10$ points and $77 \%$ had walking difficulties. The mean progression in NIS-LL of 4.8 points after 6 months of treatment was similar to that observed in the pretreatment period. Overall, 55 and $38 \%$ of patients deteriorated after 12 months with regard to disability and NIS, respectively [63]. In other clinical studies, disease progression has been observed on tafamidis, with 40-65\% experiencing neurological progression (NIS-LL $\geq 2$-point increase) at 12 months [64-66]. In a longitudinal Italian study, autonomic function also worsened in $56 \%$ of patients who had been treated for at least 6 months with tafamidis while $15 \%$ of patients showed cardiac disease progression and 30\% developed new-onset cardiomyopathy [65]. Overall, these studies highlight that tafamidis may not effectively prevent disease progression with long-term use, in particular in patients with more advanced polyneuropathy. However, more promising results have been reported from a recent Phase III study (ATTR-ACT) in patients with ATTR amyloidosis with cardiomyopathy. At 30 months, tafamidis was associated with a lower all-cause mortality compared with placebo (29.5 vs 42.9\%; hazard ratio, 0.70 ; $95 \%$ CI $0.51-$ 0.96), and a lower frequency of cardiovascular hospitalizations ( 0.48 per year vs 0.7 per year; relative risk ratio, 0.68 ; 95\% CI: 0.56-0.81). The improvement in all-cause mortality and rate of cardiovascular-related hospitalizations with tafamidis was observed in patients with wtATTR or hATTR amyloidosis, although the differences compared with placebo were not statistically significant in patients with TTR mutations. Significantly lower rates of decline in the key secondary end points of distance for 6-min walk test $(\mathrm{p}<0.001)$ and QoL (Kansas City Cardiomyopathy Questionnaire-Overall Summary score; $\mathrm{p}<0.001$ ) were also observed with tafamidis treatment. The benefit of tafamidis compared with placebo extended to exploratory end points, with trends towards improvement in $N$ terminal prohormone of brain-type natriuretic peptide (NT-proBNP) levels and echocardiographic structure and functional findings. The safety profiles of tafamidis and placebo in this study were similar, and treatment-emergent AEs in the tafamidis group were generally mild to moderate in severity [67].

\section{Diflunisal}

Diflunisal (Dolobid ${ }^{\circledR}$ ) is a generic nonsteroidal anti-inflammatory drug (NSAID) that has been repurposed as a tetramer stabilizer [68]. Although it is not approved for use in hATTR amyloidosis, it has been used off-label in some countries but is not widely available [10]. Similar to tafamidis, diflunisal has been shown to slow neuropathic progression (measured by NIS+7) relative to placebo at 24 months in one Phase III randomized, placebo-controlled study [69]. Patient QoL, measured by the Medical Outcomes Study Short-Form (36-item) questionnaire, was also improved relative to placebo. Of the patients enrolled, only $48 \%$ completed study treatment, and discontinuation was attributed largely to disease progression with a mean change in NIS +7 of +8.2 at 2 years (longitudinal intentionto-treat analysis). Diflunisal treatment of patients with cardiac involvement at baseline did not significantly reduce 
left ventricular (LV) wall thickness or LV longitudinal strain compared with placebo [70] and there is limited evidence of diflunisal efficacy in patients with cardiomyopathy from other studies [10,71]. Diflunisal was generally well tolerated in the Phase III trial, however cases of renal dysfunction and thrombocytopenia have been reported in other studies of long-term use in patients with hATTR amyloidosis [72]. The use of NSAIDs is also associated with an increased risk of GI and renal AEs, as well as cardiotoxicity [73-75], and the drugs are contraindicated in patients with congestive heart failure or renal insufficiency [10]. As a result, the use of diflunisal for the treatment of hATTR amyloidosis is limited by the cardiac involvement frequently observed in these patients.

TTR-lowering treatments

Inotersen (TEGSEDI ${ }^{\text {TM }}$ ) is an antisense oligonucleotide (ASO) therapy that was designed to suppress the expression of both WT and mutant forms of TTR. It has recently received marketing authorization approval from the European Commission (EC) for the treatment of stage 1 or stage 2 polyneuropathy in adult patients with hATTR amyloidosis [76] and regulatory approval from the US FDA for the treatment of the polyneuropathy of hATTR amyloidosis in adults [77]. These approvals were based on results from the Phase III NEURO-TTR study in patients with hATTR amyloidosis with symptoms of polyneuropathy. In this study, inotersen-treated patients experienced significant benefit compared with placebo-treated patients across both coprimary end points: the Norfolk QoL-DN questionnaire and modified NIS +7 (mNIS+7), a measure of neuropathic disease progression. However, neither end point demonstrated a mean improvement from study entry baseline with inotersen treatment, but instead a slower worsening from baseline compared with placebo treatment was observed. In addition, no significant improvements were observed in cardiac parameters in the subpopulation of patients with cardiac involvement at baseline [78]. With respect to safety, inotersen is associated with an increased risk of thrombocytopenia and glomerulonephritis requiring enhanced monitoring to support early detection and management of these risks.

Other agents in development

Other agents that are currently in late clinical development for the treatment of hATTR amyloidosis include: new TTR stabilizer molecules with higher tetramer stabilization properties (SOM0226, CSP-1103 and AG10); a combination of the antibiotic doxycycline, which disrupts TTR amyloid fibril formation, and tauroursodeoxycholic acid, which can reduce nonfibrillar TTR deposition [79,80]; and anti-TTR monoclonal antibodies that bind to amyloid monomers and inhibit fibril formation and promote the uptake of TTR aggregates by phagocytosis [81,82]. However, data from randomized controlled trials of these agents are currently lacking.

\section{Introduction to patisiran}

Patisiran $\left(\right.$ ONPATTRO $\left.^{\text {TM }}\right)$ is a first-of-its kind RNA interference (RNAi)-based therapy designed to reduce levels of both WT and mutant TTR for the treatment of patients with hATTR amyloidosis. The short interfering RNA (siRNA) of patisiran targets TTR, and is formulated in lipid nanoparticles (LNPs) that direct it to the liver which is the primary site of TTR production [83,84]. The clinical development of patisiran occurred in parallel with that of inotersen. Patisiran recently received regulatory approval from the US FDA for the treatment of the polyneuropathy of hATTR amyloidosis in adults [85], and was approved by the EC for the treatment of hATTR amyloidosis in adults with stage 1 or stage 2 polyneuropathy [86], on the basis of significant improvements in a range of clinical and QoL parameters in the Phase III APOLLO clinical trial.

\section{Rationale for patisiran development}

The rationale for the development of patisiran was based on the hypothesis that by preventing the production of liver-derived circulating mutant and WT amyloidogenic TTR protein, it is possible to reduce deposition and promote the stabilization or clearance of TTR amyloid deposits [87,88]. This can lead to stabilization or improvement of disease manifestations including polyneuropathy and cardiomyopathy, as well as improvement of overall health. Support for the strategy of lowering pathogenic protein levels comes from studies across the amyloidosis field. In a study of patients with AL amyloidosis, reduction of amyloidogenic protein levels by $50 \%$ resulted in a 5 -year survival rate of $88 \%$ compared with $39 \%$ in patients without a $50 \%$ reduction [89]. More recently, effective reduction of amyloidogenic light chain concentration, defined by stringent haematological response criteria, has been shown to translate into organ response and survival benefit [90]. Similarly, in AA amyloidosis, sustained suppression of the circulating amyloidogenic precursor serum AA is associated with favorable renal outcome and prolonged survival [91]. In addition, a higher amyloid load was associated with worse organ function deterioration in 


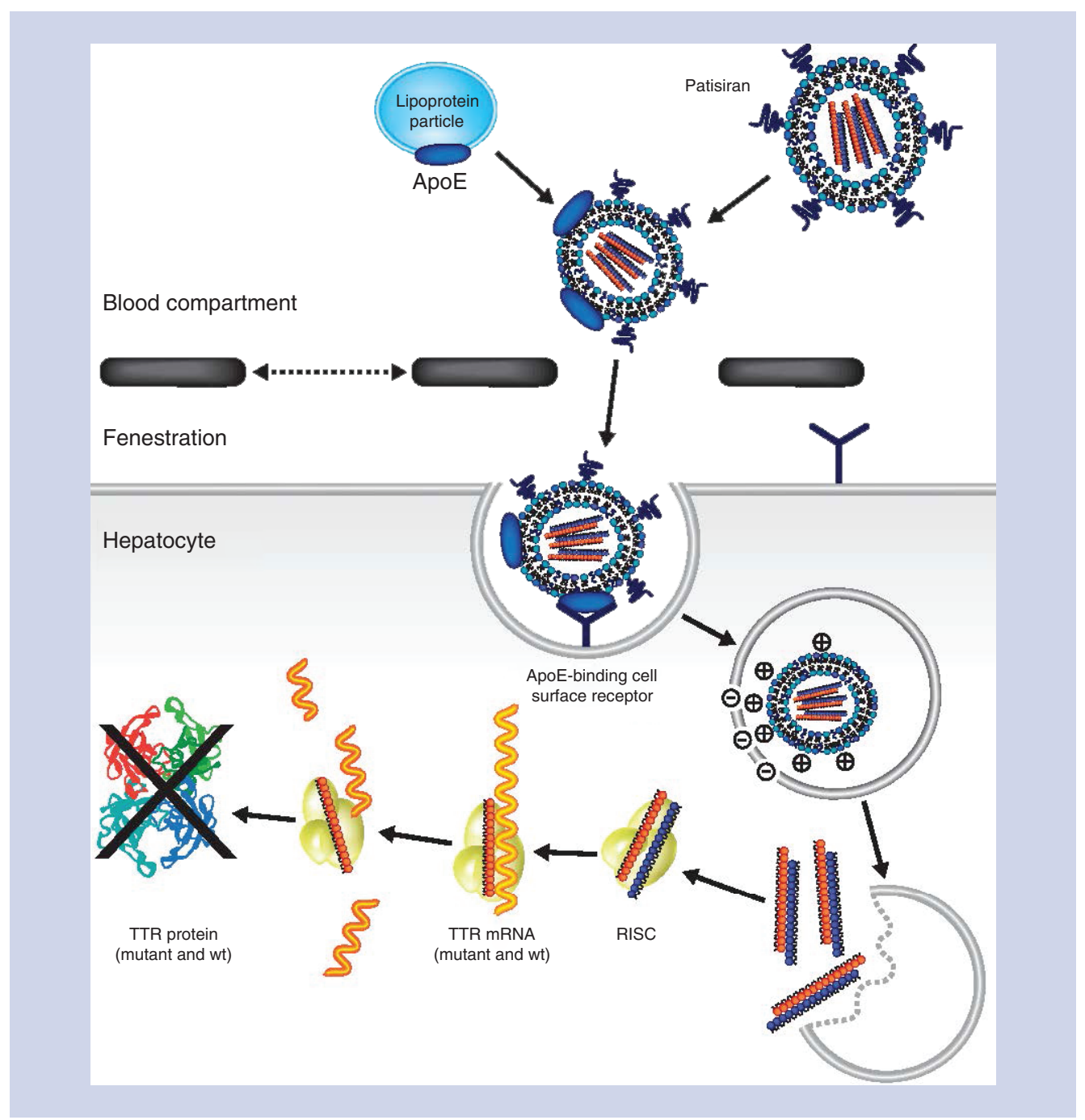

Figure 2. Mode of action of the RNA interference therapeutic patisiran. Following intravenous administration and release of the small interfering RNA (siRNA) into the cytoplasm, patisiran is loaded onto the RISC. The two strands of the siRNA duplex are then separated, and the antisense strand complexed to RISC binds specifically to the complementary sequence within the $3^{\prime}$ UTR of both mutant and WT TTR mRNA. The Argonaute-2 endonuclease within RISC then mediates mRNA cleavage, leading to knockdown of mutant and WT TTR mRNA and suppression of TTR protein production.

RISC: RNA-induced silencing complex; RNAi: RNA interference; TTR: Transthyretin; WT: Wild-type.

patients with AA amyloidosis [92]. As discussed, reduction of mutant TTR levels following liver transplantation has also been shown to slow disease progression in a select group of patients with hATTR amyloidosis with early-stage polyneuropathy $[43,93]$. The disease progression observed post-liver transplantation is primarily due to the continued deposition of WT TTR amyloid fibrils $[6,31,56]$ and therefore treatments that reduce levels of both mutant and WT TTR could overcome this deleterious effect.

\section{Mechanism of action of patisiran}

The mechanism of action of patisiran is depicted in Figure 2. RNAi is a natural endogenous intracellular catalytic mechanism for the control of gene expression that utilizes siRNAs to reduce translation of the target protein product [94]. Patisiran siRNA is designed to target a sequence within the untranslated region of TTR mRNA, conserved across WT and all TTR variants, and is therefore able to reduce both WT and pathogenic protein, regardless of the specific mutation [95]. 
Following intravenous (iv) infusion of the encapsulated patisiran siRNA, the LNPs are opsonized by apolipoprotein E (ApoE), enter the liver through vascular fenestrations and bind to the ApoE receptors on the surface of hepatocytes [95]. Subsequent endocytosis facilitates the ionization of the lipid component which fuses with the endosomal membrane, resulting in the release of patisiran siRNA into the cytoplasm. The siRNA is recognized by and loaded into the cytoplasmic enzymatic protein complex RNA-induced silencing complex (RISC), composed of Argonaute-2 (Argo-2), the ribonuclease Dicer and trans-activation responsive RNA-binding protein. The RNA duplex unwinds, and Argo-2 cleaves and discards the passenger (sense) strand. The remaining guide (antisense) strand guides the RISC complex to its complementary mRNA where it binds specifically to the conserved sequence in the 3' untranslated region of the TTR mRNA. Argo-2 within the RISC/siRNA complex then catalytically degrades WT and mutant TTR mRNA, resulting in a reduction of both WT and mutant TTR protein synthesis [94,95]. The patisiran mechanism of action is thereby distinct from that of OLT and TTR tetramer stabilizers. In addition, it is distinct in its mode of action from the ASO inotersen. Chemically synthesized ASOs bind in a 1:1 stoichiometric manner to a complementary section of mRNA, and lead to its degradation via an RNAase $\mathrm{H}$-dependent mechanism [96]. By comparison, the catalytic process of RNAi utilized by patisiran needs only a single molecule to cleave a large number of target mRNAs [97].

\section{Patisiran infusion}

Patisiran is formulated to $2 \mathrm{mg} / \mathrm{ml}(10 \mathrm{mg} / 5 \mathrm{ml})$ in LNPs, and is administered via iv infusion once every 3 weeks at a dose of $0.3 \mathrm{mg} / \mathrm{kg}$ body weight. For patients weighing $100 \mathrm{~kg}$ or more, the recommended dosage is $30 \mathrm{mg}$. Patients receive premedication consisting of dexamethasone, $\mathrm{H} 1$ and $\mathrm{H} 2$ blockers, and acetominophen/paracetamol, $60 \mathrm{~min}$ prior to patisiran dosing to minimize the risk of infusion-related reactions, which can occur with oligonucleotide therapies and LNPs. Infusion duration is approximately $80 \mathrm{~min}$, starting at a lower infusion rate of approximately $1 \mathrm{ml} / \mathrm{min}$ for the first $15 \mathrm{~min}$ before increasing to approximately $3 \mathrm{ml} / \mathrm{min}$. Patients also receive an oral daily supplement of the recommended daily allowance of vitamin A, to compensate for the expected effect of TTR lowering on vitamin A levels.

\section{Overview of clinical development}

The early clinical development programme for patisiran included two Phase I single ascending-dose studies in healthy volunteers (NCT01559077 and NCT02053454 [Japanese cohort]) and a multiple ascending-dose Phase II study in patients with hATTR amyloidosis (NCT01617967). A long-term Phase II open-label extension (OLE) study in patients who completed the Phase II study (NCT01961921) has also been completed. The pivotal, randomized, placebo-controlled Phase III study in patients with hATTR amyloidosis (APOLLO; NCT1960348) has been completed and a Global OLE study (NCT02510261) for patients who completed APOLLO or the Phase II OLE study is ongoing.

\section{Patisiran: Pharmacology}

The formulation of patisiran in LNPs distributes it predominantly to the liver. LNPs have been shown to be minimally distributed to other organs that have a fenestrated endothelium (e.g., the spleen) [98-100] although there is no evidence for penetration of the central nervous system or eye following systemic administration of such formulations [100].

Pharmacokinetic parameters of the patisiran siRNA were evaluated across all phases of clinical development. Systemic exposure to patisiran increased in an approximately dose-proportional manner over the dose ranges tested in both Phase I and II studies [95,101]. In Phase II, there was no accumulation of siRNA following the second dose and measurements of the encapsulated versus unencapsulated concentrations of TTR siRNA after each dose indicated stability of the circulating LNP formulation [101]. Patisiran is mainly cleared through metabolism by nucleases to nucleotides of various lengths and less than $1 \%$ of the administered dose is excreted unchanged in the urine [85].

The pharmacodynamic activity of patisiran was primarily evaluated based on serum TTR level, the reduction of which was rapid, robust and sustained during treatment. In the Phase I study in healthy volunteers, a single iv dose of 0.15 or $0.3 \mathrm{mg} / \mathrm{kg}$ bodyweight achieved mean serum TTR suppression of 82.3 and $86.8 \%$, respectively. Peak suppression was observed after 10 days, returning to near baseline by day 70 [95]. In the Phase II study, dose-dependent suppression of TTR was observed with $>80 \%$ suppression maintained with two consecutive doses at $0.3 \mathrm{mg} / \mathrm{kg}$ body weight given every 3 weeks ( $\mathrm{q} 3 \mathrm{w}$ ). A maximum reduction of $96 \%$ was achieved in this treatment 
group [101]. In patients with a Val30Met mutation, the level of reduction of WT and mutant TTR was equivalent and overall TTR reduction was indistinguishable between Val30Met and non-Val30Met patients. Significantly, the extent of TTR reduction was not affected in patients taking tafamidis or diflunisal, suggesting that these TTR stabilizer drugs do not interfere with the pharmacological activity of patisiran [101]. In the Phase II OLE and the Phase III APOLLO studies, treatment with patisiran $0.3 \mathrm{mg} / \mathrm{kg}$ body weight $\mathrm{q} 3 \mathrm{w}$ resulted in a reduction in serum TTR of approximately $80 \%$ by 2 weeks after the first dose, which was sustained across 24 and 18 months of the respective studies $[102,103]$. In the Phase II OLE and Phase III studies, a correlation was observed between the degree of serum TTR reduction and the change in mNIS+7 (measure of polyneuropathy, but slightly different scoring system from the mNIS+7 utilized in the inotersen Phase III study), supporting the therapeutic hypothesis for patisiran [102,104].

Across all studies, trends towards lowering retinol-binding protein (RBP) and vitamin A were consistent with those seen for total serum TTR, which was expected based on the known role of TTR in binding and transport of the RBP-vitamin A complex $[95,101,102]$.

\section{Patisiran: Clinical efficacy}

Phase II \& Phase II OLE

The Phase II study was conducted to evaluate the safety, tolerability and pharmacokinetic/pharmacodynamic profile of multiple ascending doses $(0.01,0.05$ and $0.3 \mathrm{mg} / \mathrm{kg}$ body weight) of patisiran in 26 patients with hATTR amyloidosis with polyneuropathy [101]. The majority of patients enrolled (76\%) had the Val30Met TTR mutation. Walking difficulties requiring the use of a crutch were present in $14 \%$ of the patients with the remainder having unimpaired ambulation. Concurrent stabilizer use was common, with $48 \%$ of patients receiving tafamidis and $24 \%$ receiving diflunisal.

The Phase II study identified patisiran $0.3 \mathrm{mg} / \mathrm{kg}$ body weight $\mathrm{q} 3 \mathrm{w}$ as the optimal dosage and schedule to administer in an OLE study, which monitored the long-term safety and efficacy of patisiran. Eligible patients had biopsy-proven hATTR amyloidosis with mild-to-moderate neuropathy, and had previously received and tolerated patisiran in the Phase II study [103]. Of the 27 patients enrolled, 12 (44\%) had walking difficulties and 11 (41\%) were included in the cardiac subgroup. Improvement or stabilization of polyneuropathy, measured by mNIS +7 , was reported in $20 / 27$ patients $(74 \%)$ at 24 months, with an overall mean decrease (improvement) in mNIS+7 from baseline of 6.95 points [105]. Echocardiographic measures and cardiac biomarkers were stable throughout 24 months in the cardiac subgroup. Improvements in nerve-fibre density and a corresponding reduction in amyloid burden in skin biopsies over 24 months provided preliminary evidence that patisiran may halt or reverse neuropathy.

\section{Phase III APOLLO}

To date, APOLLO is the largest randomized, double-blind, placebo-controlled, Phase III study conducted in patients with hATTR amyloidosis [106]. The study included a range of end points to capture the diverse symptoms of hATTR amyloidosis, and better quantify the impact of patisiran on disease burden. The primary end point was mNIS +7, a composite measure that was specifically designed to assess polyneuropathy in this disease. The key secondary end point was the effect of patisiran on QoL, as measured by the Norfolk QoL-DN 35-item questionnaire, which has been shown to correlate with disease status in patients with hATTR amyloidosis [107].

A total of 225 patients from 19 countries were randomly assigned (2:1) to receive patisiran $0.3 \mathrm{mg} / \mathrm{kg}$ body weight $(\mathrm{n}=148)$ or placebo $(\mathrm{n}=77)$, administered by iv infusion $\mathrm{q} 3 \mathrm{w}$ for 18 months. Baseline demographics and clinical characteristics were generally balanced between the two treatment groups, although the Val30Met mutation was more prevalent in the placebo group compared with the patisiran group (52 vs 38\%). Overall, 126 patients ( $56 \% ; 47 \%$ placebo vs $61 \%$ patisiran) fulfilled the criteria to be included in a prespecified cardiac subpopulation (baseline LV wall thickness $\geq 13 \mathrm{~mm}$ and no history of aortic valve disease or hypertension). In the modified ITT population (patients who had received at least one dose of patisiran or placebo), 138 (93\%) patisiran-treated patients completed the study compared with $55(71 \%)$ patients in the placebo group.

Key efficacy outcomes in the total population are summarized in Table 1. For the primary end point, patisiran treatment led to a significant improvement in polyneuropathy versus placebo (least-squares mean [LSM] difference in mNIS+7; -34 points; $\mathrm{p}<0.001$ ) (Figure 3). At 18 months, patients receiving patisiran also experienced improvement in neuropathy compared with baseline, whereas those on placebo had worsened neuropathy at this timepoint (Table 1) [102]. 


\begin{tabular}{|c|c|c|c|c|}
\hline End point in mITT population* & $\begin{array}{l}\text { Placebo } \\
(\mathrm{N}=77)\end{array}$ & $\begin{array}{l}\text { Patisiran } \\
(\mathrm{N}=148)\end{array}$ & $\begin{array}{l}\text { LS mean treatment } \\
\text { difference } \\
\text { (patisiran-placebo) }\end{array}$ & $\begin{array}{l}\mathrm{p} \text { value for } \\
\text { treatment } \\
\text { difference }\end{array}$ \\
\hline \multicolumn{5}{|l|}{ Primary end point } \\
\hline $\begin{array}{l}\text { Modified Neuropathy Impairment Score }+7 \text { : } \\
\text { - Mean (SD) baseline score } \\
\text { - LS mean change from baseline at } 18 \text { months (SEM) }\end{array}$ & $\begin{array}{l}74.6(37.0) \\
+28.0(2.6)\end{array}$ & $\begin{array}{l}80.9(41.5) \\
-6.0(1.7)\end{array}$ & $-34.0(3.0)$ & $<0.001$ \\
\hline \multicolumn{5}{|l|}{ Secondary end points } \\
\hline $\begin{array}{l}\text { Norfolk QoL-DN: } \\
\text { - Mean (SD) baseline score } \\
\text { - LS mean change from baseline at } 18 \text { months (SEM) }\end{array}$ & $\begin{array}{l}55.5(24.3) \\
14.4(2.7)\end{array}$ & $\begin{array}{l}59.6(28.2) \\
-6.7(1.8)\end{array}$ & $-21.1(3.1)$ & $<0.001$ \\
\hline $\begin{array}{l}\text { NIS-W: } \\
- \text { Mean (SD) baseline score } \\
\text { - LS mean change from baseline at } 18 \text { months }\left(\text { SEM) }{ }^{\dagger}\right.\end{array}$ & $\begin{array}{l}29.0(23.0) \\
17.9(2.0)\end{array}$ & $\begin{array}{l}32.7(25.2) \\
0.1(1.3)\end{array}$ & $-17.9(2.3)$ & $<0.001$ \\
\hline $\begin{array}{l}\text { R-ODS: } \\
\text { - Mean (SD) baseline score } \\
\text { - LS mean change from baseline at } 18 \text { months (SEM) })^{\ddagger}\end{array}$ & $\begin{array}{l}29.8(10.8) \\
-8.9(0.9)\end{array}$ & $\begin{array}{l}29.7(11.5) \\
0.0(0.6)\end{array}$ & $9.0(1.0)$ & $<0.001$ \\
\hline $\begin{array}{l}\text { 10-m walk test, } \mathrm{m} / \mathrm{s} \text { : } \\
- \text { Mean (SD) baseline score } \\
- \text { LS mean change from baseline at } 18 \text { months (SEM) }{ }^{\S}\end{array}$ & $\begin{array}{l}0.79(0.32) \\
-0.24(0.04)\end{array}$ & $\begin{array}{l}0.80(0.40) \\
0.08(0.02)\end{array}$ & $0.31(0.04)$ & $<0.001$ \\
\hline $\begin{array}{l}\mathrm{mBMI}-\mathrm{BMI} \mathrm{kg} / \mathrm{m}^{2} \times \text { albumin, } \mathrm{g} / \mathrm{dl}: \\
- \text { Mean (SD) baseline score } \\
- \text { LS mean change from baseline at } 18 \text { months (SEM) } \mathbb{I}\end{array}$ & $\begin{array}{l}989.9(214.2) \\
-119.4(14.5)\end{array}$ & $\begin{array}{l}969.7(210.5) \\
-3.7(9.6)\end{array}$ & $115.7(16.9)$ & $<0.001$ \\
\hline $\begin{array}{l}\text { COMPASS-31: } \\
\text { - Mean (SD) baseline score } \\
\text { - LS mean change from baseline at } 18 \text { months }(\text { SEM })^{\#}\end{array}$ & $\begin{array}{l}30.3(16.4) \\
2.2(1.9)\end{array}$ & $\begin{array}{l}30.6(17.6) \\
-5.3(1.3)\end{array}$ & $-7.5(2.2)$ & $<0.001$ \\
\hline \multicolumn{5}{|l|}{ Exploratory end points (cardiac subpopulation) ${ }^{\star \star}$} \\
\hline No. of patients & 36 & 90 & & \\
\hline $\begin{array}{l}\text { Mean left ventricular wall thickness, } \mathrm{cm} \text { : } \\
\text { - Baseline, mean (SD) } \\
\text { - LS mean change from baseline at } 18 \text { months (SEM) }\end{array}$ & $\begin{array}{l}1.64(0.21) \\
-0.007(0.03)\end{array}$ & $\begin{array}{l}1.68(0.26) \\
-0.10(0.02)\end{array}$ & $-0.093(0.039)$ & 0.017 \\
\hline $\begin{array}{l}\text { Longitudinal strain, } \% \text { : } \\
\text { - Baseline, mean (SD) } \\
\text { - LS mean change from baseline at } 18 \text { months (SEM) }\end{array}$ & $\begin{array}{l}-15.66(3.51) \\
1.46(0.48)\end{array}$ & $\begin{array}{l}-15.13(3.41) \\
0.08(0.28)\end{array}$ & $-1.37(0.56)$ & 0.015 \\
\hline $\begin{array}{l}\text { NT-proBNP, pg/ml }{ }^{\dagger \dagger:} \\
\text { - Baseline, geometric mean (CV\%) } \\
\text { - Fold change from baseline at } 18 \text { months } \neq \ddagger\end{array}$ & $\begin{array}{l}711.1(151.08) \\
1.97\end{array}$ & $\begin{array}{l}726.9(103.02) \\
0.89\end{array}$ & $0.45^{\S \S}$ & $<0.001$ \\
\hline \multicolumn{5}{|c|}{ 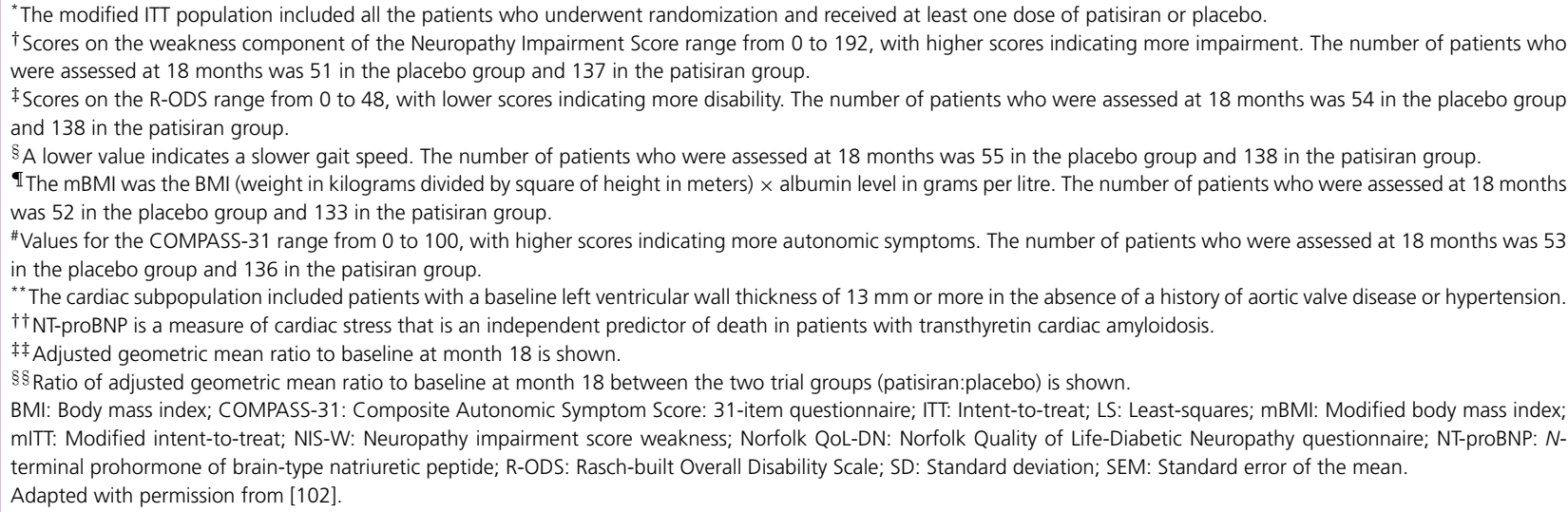 } \\
\hline
\end{tabular}

Statistically significant improvements were also observed for QoL outcomes, including the Norfolk QoL-DN score (LSM difference vs placebo at 18 months: -21 points; $\mathrm{p}<0.001$ ) (Figure 4). Notably, at 18 months, 56 and $51 \%$ of patisiran-treated patients experienced an improvement in mNIS+7 and Norfolk QoL-DN scores from baseline, respectively, compared with 4 and $10 \%$ of placebo-treated patients. Improvements in mNIS+7 and Norfolk QoL-DN scores with patisiran versus placebo were observed across all subgroups evaluated (age, gender, race, genotype, FAP stage, previous stabilizer use and cardiac involvement) [102]. Significant improvements in favor of patisiran were observed for all other secondary end points, including measures of activities of daily 


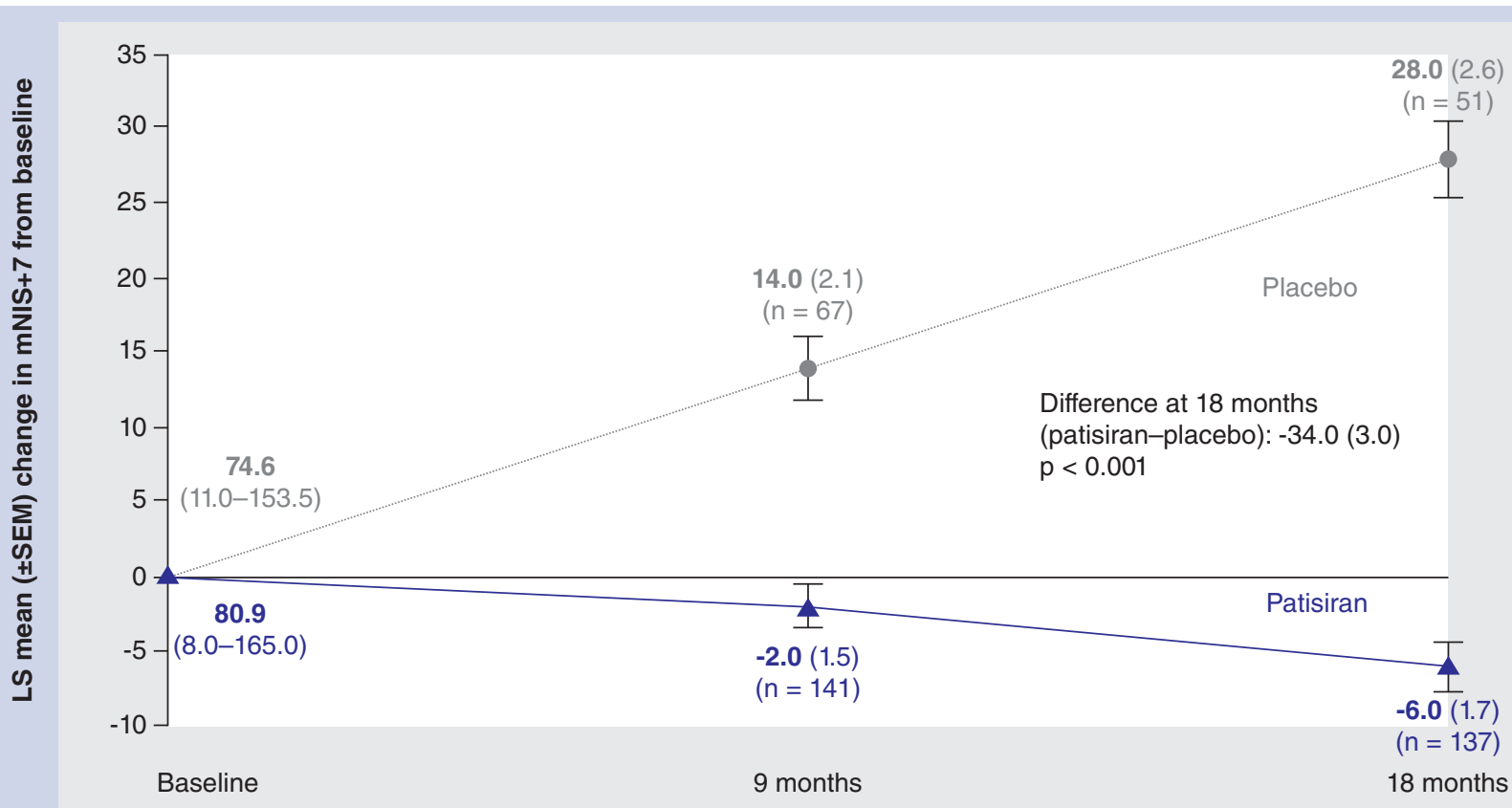

Figure 3. LS mean (SEM) change in the mNIS+7 over time. LS mean (SEM) changes from baseline in mNIS+7 at 9 and 18 months are indicated above the graph. At baseline, mean (range) is shown for each treatment group. Reduction in mNIS +7 score represents improvement.

LS: Least-squares; mNIS + 7: Modified Neuropathy Impairment Score +7; SEM: Standard error of the mean.

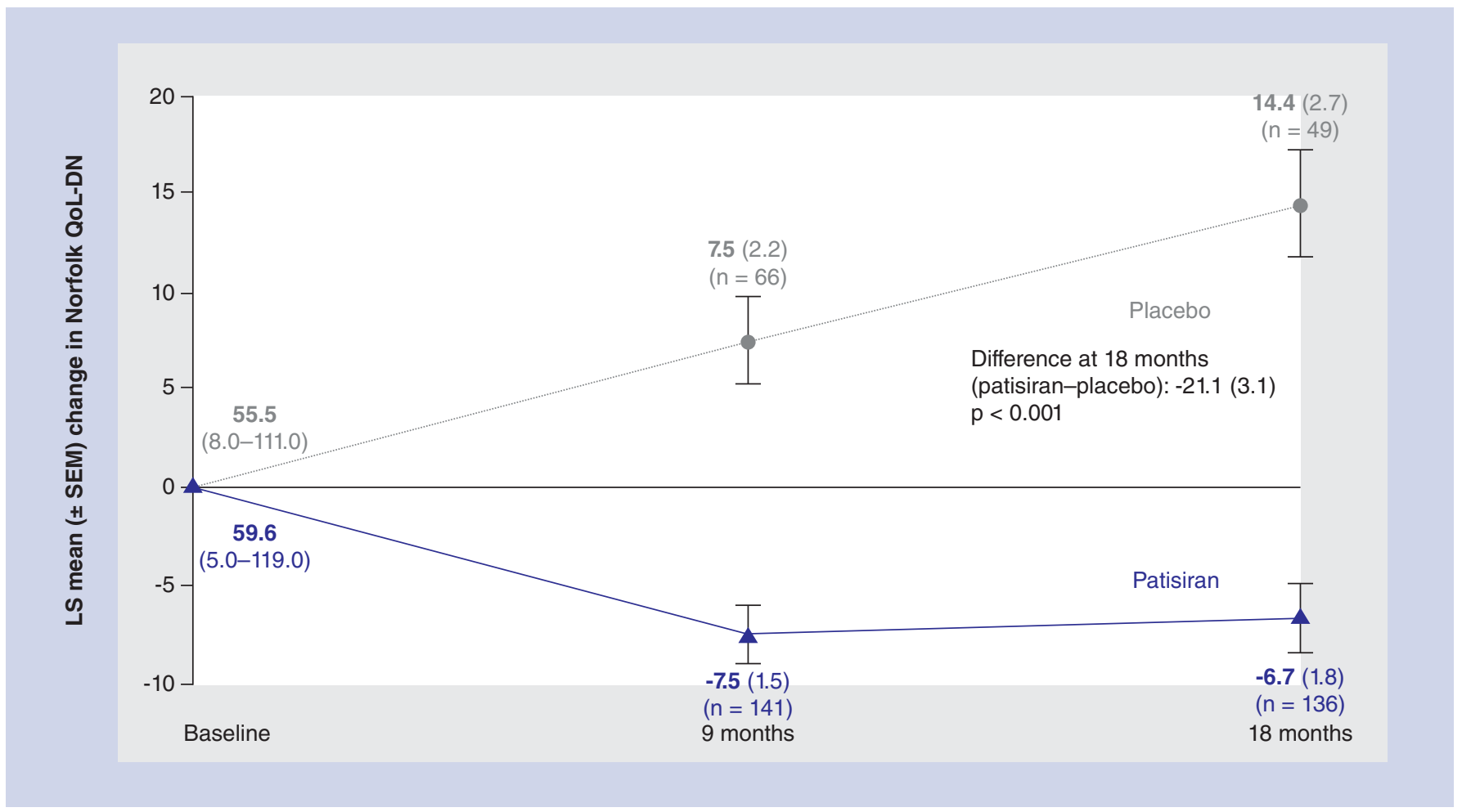

Figure 4. LS mean (SEM) change in Norfolk QoL-DN scores over time in the patisiran and placebo groups. LS mean (SEM) changes from baseline in Norfolk QoL-DN at 9 and 18 months are indicated above the graph. At baseline, mean (range) is shown for each treatment group. Reduction in Norfolk QoL-DN score represents improvement.

LS: Least-squares; Norfolk QoL-DN: Norfolk Quality of Life-Diabetic Neuropathy score; SEM: standard error of the mean. 
living, walking ability, nutritional status and autonomic symptoms (Table 1). Of particular significance were improvements from baseline in 53 and $40 \%$ of patisiran-treated patients in gait speed (10-meter walk) and motor strength, respectively, compared with 13 and $1 \%$ of placebo-treated patients, indicating a benefit of patisiran on physical functioning. PND score, indicative of neuropathy stage and ambulation status, was stable or improved from baseline at 18 months in 65 and $8 \%$ of patisiran-treated patients compared with 30 and $0 \%$ in the placebo group. In the cardiac subpopulation, patisiran treatment was associated with improvement in cardiac structure and function compared with placebo, with significant reductions in LV wall thickness (LSM difference \pm standard error of the mean $[S E M]-0.09 \pm 0.04 \mathrm{~cm} ; \mathrm{p}=0.017$ ) and LV longitudinal strain (LSM difference \pm SEM $-1.37 \pm 0.56 \% ; \mathrm{p}=0.015)$ at 18 months $[102,108]$. A greater proportion of patisiran-treated patients had a reduction from baseline in mean $\mathrm{LV}$ wall thickness $>2 \mathrm{~mm}$ compared with placebo ( $29.1 \mathrm{vs} 4.0 \%$, respectively) or an absolute decrease from baseline in global longitudinal strain of $>2 \%(21.3 \mathrm{vs} 8.0 \%)$ [108], with these thresholds chosen to represent clinically relevant changes based on assessments in patients with ATTR or AL amyloidosis [109]. In addition, patisiran treatment reduced NT-proBNP levels compared with placebo at 18 months, with a ratio of fold change between the treatment groups of $0.45(\mathrm{p}<0.001)$, representing a $55 \%$ difference in favor of patisiran [102].

\section{Patisiran: safety \& tolerability}

Patisiran was generally well tolerated and showed a consistent safety profile in patients with hATTR amyloidosis, with a low rate of treatment discontinuations due to AEs across clinical studies. In the Phase II study, one patient withdrew due to four serious AEs ([SAEs] urinary tract infection, sepsis, nausea and vomiting) and an additional SAE of extravasation-related cellulitis was reported [101]. In the Phase II OLE there were no discontinuations due to AEs, drug-related severe AEs, SAEs or deaths [110]. In APOLLO, AEs leading to discontinuation occurred less frequently with patisiran than placebo ( 5 vs $14 \%$ ), with cardiac failure leading to discontinuation in two patients ( $1 \%$ of patisiran group). The frequency of severe AEs and SAEs in APOLLO were similar between the patisiranand placebo-treatment groups (Table 2), as was the number of deaths (patisiran, $\mathrm{n}=7$ [4.7\%]; placebo, $\mathrm{n}=6$ [7.8\%]) [102]. No deaths were considered related to study drug. In the ongoing Global OLE study of patisiran $(\mathrm{n}=189)$, AEs led to withdrawal of 16 patients, and two patients had a drug-related SAE; one patient with phlebitis secondary to drug extravasation, cellulitis and hypotension; one patient with abdominal discomfort who withdrew from the study. To date there have been 11 deaths in this study, none of which was considered related to study drug [110]. Full safety data for the individual studies have been previously reported [95,101,102].

Across the patisiran clinical programme, the majority of AEs were mild to moderate in intensity. The most common AE related to study drug in the Phase II study was infusion-related reactions (IRRs), which occurred in $3 / 29(10.3 \%)$ patients in the ITT population [101]. IRRs were also the most common AE related to study drug in the 24-month Phase II OLE study (22\%) [105], and have been reported in 10\% of patients to date in the Global OLE study [110]. In APOLLO, IRRs (19 vs 9\%) and peripheral edema (30 vs 22\%) occurred more frequently with patisiran than placebo; IRRs decreased in frequency over time. No clinically relevant changes in laboratory values related to patisiran were observed in the APOLLO study, including platelet levels, and liver or kidney function tests [102]. In addition, there were no safety signals regarding cataracts, hyperglycemia, infection or osteopenia/osteoporosis for patisiran [111].

Although TTR has a role in transporting thyroxine and the RBP-vitamin A complex, no significant decline in thyroid function and no clinical manifestations of vitamin A deficiency have been observed in the patisiran clinical programme to date as a result of the lowered TTR, RBP and vitamin A levels [95,101,102].

The tolerability and efficacy of patisiran contributed to the high retention rate in the APOLLO study, and 99\% of eligible patients have subsequently enrolled in the ongoing OLE study $(\mathrm{n}=211)$.

\section{Patisiran: regulatory affairs}

On 10 August 2018, patisiran was granted approval by the FDA for the treatment of the polyneuropathy of hATTR amyloidosis in adults [85]. On 30 August 2018, patisiran received approval from the EC for the treatment of hATTR amyloidosis in adults with stage 1 or stage 2 polyneuropathy. The recommended Summary of Product Characteristics for the European Union includes data on secondary and exploratory end points [86]. 
Table 2. Safety and tolerability in Phase III APOLLO study.

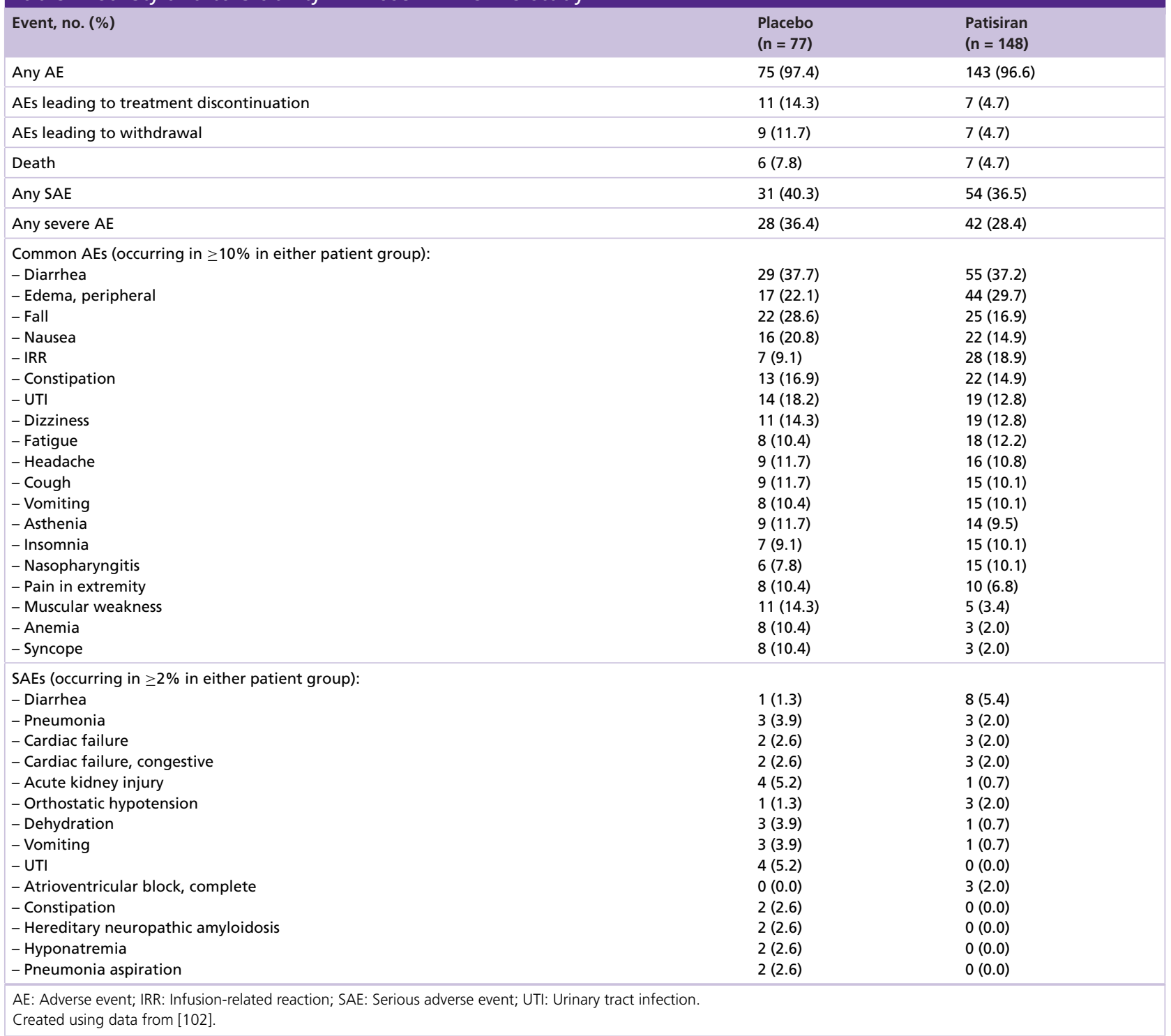

\section{Conclusion}

hATTR amyloidosis is a rapidly progressive, life-threatening disease with symptoms of peripheral sensorimotor neuropathy, autonomic neuropathy and/or cardiomyopathy. The heterogenous nature of the disease has presented challenges to diagnosis, and the efficacy of available therapies has been limited to certain patient populations.

The patisiran clinical programme has demonstrated that TTR reduction is associated with improvement in clinical outcomes across a broad population of patients with hATTR amyloidosis [95,101,102]. Notably, the 7.0- and 6.0-point improvements in the polyneuropathy measure mNIS+7 in the Phase II OLE and Phase III APOLLO trials, respectively, represent a marked contrast to the expected 17.8-point worsening estimated from a population with similar baseline neuropathy scores in a natural history study [8]. In APOLLO, patisiran also significantly improved autonomic neuropathy, patient QoL end points and some cardiac parameters, compared with placebo and/or baseline. The impact of patisiran treatment on the diverse manifestations of hATTR amyloidosis compares favorably to TTR stabilizers, which have not been shown to halt or reverse neurological or cardiac decline associated with hATTR amyloidosis. Direct comparison of the Phase III trials of TTR-lowering compounds patisiran and inotersen may be limited by the varying sample sizes, multiple different TTR mutations and the use of different mNIS+7 scales. However, patisiran is unique as the only approved/available treatment that has improved polyneuropathy. Patisiran was well tolerated and has demonstrated a manageable safety profile in patients 
with hATTR amyloidosis, with a low rate of treatment discontinuations due to AEs across the clinical development programme. To date, there have been no observed AEs resulting from the administration of steroid pretreatment, although the effects of steroid use typically manifest after many years and therefore long-term monitoring for the chronic effects of steroids may be advisable.

The future clinical development of patisiran may include strategies to improve the patient treatment experience. In the ongoing Phase III OLE, patients in selected regions, where local and country regulations allowed, have received home infusions by a health care professional. Home infusions were allowed following three completed doses at a clinical site with no evidence of IRRs or other AEs, and preliminary data suggest this strategy may be viable in the future [112]. Furthermore, patients may have a preference for subcutaneous over iv delivery due to the advantages of self-administration, greater mobility for patients and potential reduction in IRRs. A Phase I study of a subcutaneously administered investigational RNAi (ALN-TTRsc02) for the treatment of ATTR amyloidosis has demonstrated the potential of a 3-monthly injection schedule, and warrants continued evaluation in larger cohorts of patients [113].

Based on the positive results from APOLLO, patisiran was recently granted regulatory approval by the FDA and the EC, marking the start of a new class of drugs that harness the endogenous mechanism of RNAi to reduce production of a disease-causing protein. Patisiran functions upstream of gene transcription, setting it apart from existing drug classes, such as small molecules and antibody-based therapeutics. With the increasing number of molecules in development that have different modes of action, the potential for future management of hATTR amyloidosis involving combination therapy of patisiran with TTR stabilizers or monoclonal antibodies is an intriguing prospect.

Although the evolution of improved pharmacotherapies for the treatment of hATTR amyloidosis is encouraging, there remains a lack of therapies for ocular and leptomeningeal involvement. The rapidly progressive nature of the disease highlights the importance of an accurate and early diagnosis of hATTR amyloidosis, thus allowing patients and their affected offspring to receive the appropriate care or therapy as early as possible in the disease course.

Financial \& competing interests disclosure

AV Kristen, S Ajroud-Driss, P Gorevic and T Kyriakides have nothing to disclose. I Conceição has received financial support as a primary investigator, serving on the scientific advisory board for Alnylam Pharmaceuticals, lonis Pharmaceuticals and Pfizer. She also serves on the THAOS scientific advisory board. L Obici has received speaker fees and participated on scientific advisory boards for Alnylam Pharmaceuticals, Akcea and Pfizer. The authors have no other relevant affiliations or financial involvement with any organization or entity with a financial interest in or financial conflict with the subject matter or materials discussed in the manuscript apart from those disclosed.

The authors received editorial support from Adelphi Communications Ltd, funded by Alnylam Pharmaceuticals.

\section{References}

Papers of special note have been highlighted as: • of interest; $\bullet \bullet$ of considerable interest

1 Chiti F, Dobson CM. Protein misfolding, amyloid formation, and human disease: a summary of progress over the last decade. Annu. Rev. Biochem. 86, 27-68 (2017).

2 Shin SC, Robinson-Papp J. Amyloid neuropathies. Mt. Sinai J. Med. 79(6), 733-748 (2012).

3 Sipe JD, Benson MD, Buxbaum JN et al. Amyloid fibril proteins and amyloidosis: chemical identification and clinical classification International Society of Amyloidosis 2016 Nomenclature Guidelines. Amyloid 23(4), 209-213 (2016).

4 Perfetto F, Moggi-Pignone A, Livi R, Tempestini A, Bergesio F, Matucci-Cerinic M. Systemic amyloidosis: a challenge for the rheumatologist. Nat. Rev. Rheumatol. 6(7), 417-429 (2010).

5 Matsuda M, Gono T, Morita H, Katoh N, Kodaira M, Ikeda S. Peripheral nerve involvement in primary systemic AL amyloidosis: a clinical and electrophysiological study. Eur. J. Neurol. 18(4), 604-610 (2011).

6 Ando Y, Coelho T, Berk JL et al. Guideline of transthyretin-related hereditary amyloidosis for clinicians. Orphanet. J. Rare Dis. 8, 31 (2013).

- Overview of hereditary transthyretin-mediated (hATTR) amyloidosis, covering signs and symptoms, diagnosis and management of this disease.

7 Westermark P, Sletten K, Johansson B, Cornwell GG 3rd. Fibril in senile systemic amyloidosis is derived from normal transthyretin. Proc. Natl Acad. Sci. USA 87(7), 2843-2845 (1990).

8 Adams D, Coelho T, Obici L et al. Rapid progression of familial amyloidotic polyneuropathy: a multinational natural history study. Neurology 85(8), 675-682 (2015). 
9 Hanna M. Novel drugs targeting transthyretin amyloidosis. Curr. Heart Fail. Rep. 11(1), 50-57 (2014).

- Review of current treatment options in ATTR amyloidosis.

10 Hawkins PN, Ando Y, Dispenzeri A, Gonzalez-Duarte A, Adams D, Suhr OB. Evolving landscape in the management of transthyretin amyloidosis. Ann. Med. 47(8), 625-638 (2015).

-• Summary of the disease characteristics and management of ATTR amyloidosis. Both the neurologic and cardiac aspects of the disease are covered.

11 Kelly JW. Amyloid fibril formation and protein misassembly: a structural quest for insights into amyloid and prion diseases. Structure 5(5), 595-600 (1997).

12 Damy T, Judge DP, Kristen AV, Berthet K, Li H, Aarts J. Cardiac findings and events observed in an open-label clinical trial of tafamidis in patients with non-Val30Met and non-Val122Ile hereditary transthyretin amyloidosis. J. Cardiovasc. Transl. Res. 8(2), 117-127 (2015).

13 Ando Y, Nakamura M, Araki S. Transthyretin-related familial amyloidotic polyneuropathy. Arch. Neurol. 62(7), 1057-1062 (2005).

14 Pfizer. Recognizing TTR-FAP: a rare disease with a global presence (2014). www.recognizingttr-fap.com/epidemiology

15 Sousa A, Coelho T, Barros J, Sequeiros J. Genetic epidemiology of familial amyloidotic polyneuropathy (FAP)-type I in Povoa do Varzim and Vila do Conde (north of Portugal). Am. J. Med. Genet. 60(6), 512-521 (1995).

16 Parman Y, Adams D, Obici L et al. Sixty years of transthyretin familial amyloid polyneuropathy (TTR-FAP) in Europe: where are we now? A European network approach to defining the epidemiology and management patterns for TTR-FAP. Curr. Opin. Neurol. 29(Suppl. 1), S3-S13 (2016).

17 Vieira M, Saraiva MJ. Transthyretin: a multifaceted protein. Biomol. Concepts 5(1), 45-54 (2014).

18 Liz MA, Mar FM, Franquinho F, Sousa MM. Aboard transthyretin: from transport to cleavage. IUBMB. Life 62(6), 429-435 (2010).

19 Monaco HL, Rizzi M, Coda A. Structure of a complex of two plasma proteins: transthyretin and retinol-binding protein. Science 268(5213), 1039-1041 (1995).

20 Mangione PP, Verona G, Corazza A et al. Plasminogen activation triggers transthyretin amyloidogenesisin vitro. J. Biol. Chem. (2018) (In press).

21 Klimtchuk ES, Prokaeva T, Frame NM et al. Unusual duplication mutation in a surface loop of human transthyretin leads to an aggressive drug-resistant amyloid disease. Proc. Natl Acad. Sci. USA 115(28), E6428-E6436 (2018).

22 Benson MD. Pathogenesis of transthyretin amyloidosis. Amyloid 19(Suppl. 1), 14-15 (2012).

23 Coelho T, Maurer MS, Suhr OB. THAOS - The Transthyretin Amyloidosis Outcomes Survey: initial report on clinical manifestations in patients with hereditary and wild-type transthyretin amyloidosis. Curr. Med. Res. Opin. 29(1), 63-76 (2013).

24 Suhr OB, Lundgren E, Westermark P. One mutation, two distinct disease variants: unravelling the impact of transthyretin amyloid fibril composition. J. Intern. Med. 281(4), 337-347 (2017).

25 Dungu JN, Anderson LJ, Whelan CJ, Hawkins PN. Cardiac transthyretin amyloidosis. Heart 98(21), 1546-1554 (2012).

- Review of cardiac manifestations and their recognition in ATTR amyloidosis.

26 Maurer MS, Hanna M, Grogan M et al. Genotype and phenotype of transthyretin cardiac amyloidosis: THAOS (transthyretin amyloid outcome survey). J. Am. Coll. Cardiol. 68(2), 161-172 (2016).

27 Rowczenio DM, Noor I, Gillmore JD et al. Online registry for mutations in hereditary amyloidosis including nomenclature recommendations. Hum. Mutat. 35(9), E2403-E2412 (2014).

28 Saraiva MJ. Transthyretin mutations in hyperthyroxinemia and amyloid diseases. Hum. Mutat. 17(6), 493-503 (2001).

29 Rapezzi C, Quarta CC, Obici L et al. Disease profile and differential diagnosis of hereditary transthyretin-related amyloidosis with exclusively cardiac phenotype: an Italian perspective. Eur. Heart J. 34(7), 520-528 (2013).

30 Saraiva MJ, Birken S, Costa PP, Goodman DS. Amyloid fibril protein in familial amyloidotic polyneuropathy, Portuguese type. Definition of molecular abnormality in transthyretin (prealbumin). J. Clin. Invest. 74(1), 104-119 (1984).

31 Ruberg FL, Berk JL. Transthyretin (TTR) cardiac amyloidosis. Circulation 126(10), 1286-1300 (2012).

32 Gorevic PD, Prelli FC, Wright J, Pras M, Frangione B. Systemic senile amyloidosis. Identification of a new prealbumin (transthyretin) variant in cardiac tissue: immunologic and biochemical similarity to one form of familial amyloidotic polyneuropathy. J. Clin. Invest. 83(3), 836-843 (1989).

33 Wixner J, Mundayat R, Karayal ON et al. THAOS: gastrointestinal manifestations of transthyretin amyloidosis - common complications of a rare disease. Orphanet. J. Rare Dis. 9, 61 (2014).

34 Conceicao I, Gonzalez-Duarte A, Obici L et al. "Red-flag" symptom clusters in transthyretin familial amyloid polyneuropathy. J. Peripher. Nerv. Syst. 21(1), 5-9 (2016).

35 Mohty D, Damy T, Cosnay P et al. Cardiac amyloidosis: updates in diagnosis and management. Arch. Cardiovasc. Dis. 106(10), 528-540 (2013).

36 Adams D, Suhr OB, Hund E et al. First European consensus for diagnosis, management, and treatment of transthyretin familial amyloid polyneuropathy. Curr. Opin. Neurol. 29(Suppl. 1), S14-S26 (2016). 
-• European consensus report for diagnosis, management and treatment of hATTR amyloidosis with polyneuropathy.

37 Conceicao I, De Carvalho M. Clinical variability in type I familial amyloid polyneuropathy (Val30Met): comparison between late- and early-onset cases in Portugal. Muscle Nerve 35(1), 116-118 (2007)

38 Adams D, Theaudin M, Cauquil C, Algalarrondo V, Slama M. FAP neuropathy and emerging treatments. Curr. Neurol. Neurosci. Rep. 14(3), 435 (2014).

39 Plante-Bordeneuve V, Ferreira A, Lalu T et al. Diagnostic pitfalls in sporadic transthyretin familial amyloid polyneuropathy (TTR-FAP). Neurology 69(7), 693-698 (2007).

40 Gillmore JD, Maurer MS, Vest J et al. Analysis of disease progression in patients with transthyretin cardiac amyloidosis. Orphanet. J. Rare Dis. 10(Suppl. 1), 13 (2015).

41 Coutinho P, DeSilva A, Lima J, Barbosa A. Forty years of experience with type I amyloid neuropathy: review of 483 cases. In: Amyloid and Amyloidosis. Glenner G, Costa P, de Freitas A (Eds). Excerpta Medica, Amsterdam, The Netherlands, 88-98 (1980).

42 Koike H, Tanaka F, Hashimoto R et al. Natural history of transthyretin Val30Met familial amyloid polyneuropathy: analysis of late-onset cases from non-endemic areas. J. Neurol. Neurosurg. Psychiatry 83(2), 152-158 (2012).

43 Adams D. Recent advances in the treatment of familial amyloid polyneuropathy. Ther. Adv. Neurol. Disord. 6(2), 129-139 (2013).

44 Hurst JW, Morris DC, Alexander RW. The use of the New York Heart Association's classification of cardiovascular disease as part of the patient's complete problem list. Clin. Cardiol. 22(6), 385-390 (1999).

45 Ruberg FL, Maurer MS, Judge DP et al. Prospective evaluation of the morbidity and mortality of wild-type and V122I mutant transthyretin amyloid cardiomyopathy: the Transthyretin Amyloidosis Cardiac Study (TRACS). Am. Heart J. 164(2), 222-228 e221 (2012).

46 Castano A, Drachman BM, Judge D, Maurer MS. Natural history and therapy of TTR-cardiac amyloidosis: emerging disease-modifying therapies from organ transplantation to stabilizer and silencer drugs. Heart Fail. Rev. 20(2), 163-178 (2015).

47 Gertz MA, Kyle RA, Thibodeau SN. Familial amyloidosis: a study of 52 North American-born patients examined during a 30-year period. Mayo Clin. Proc. 67(5), 428-440 (1992).

48 Sattianayagam PT, Hahn AF, Whelan CJ et al. Cardiac phenotype and clinical outcome of familial amyloid polyneuropathy associated with transthyretin alanine 60 variant. Eur. Heart J. 33(9), 1120-1127 (2012).

49 Swiecicki PL, Zhen DB, Mauermann ML et al. Hereditary ATTR amyloidosis: a single-institution experience with 266 patients. Amyloid 22(2), 123-131 (2015).

50 Chakraborty R, Muchtar E, Gertz MA. Newer therapies for amyloid cardiomyopathy. Curr. Heart Fail. Rep. 13(5), 237-246 (2016).

51 Ericzon BG, Wilczek HE, Larsson $\mathrm{M}$ et al. Liver transplantation for hereditary transthyretin amyloidosis: after 20 years still the best therapeutic alternative? Transplantation 99(9), 1847-1854 (2015).

52 Herlenius G, Wilczek HE, Larsson M, Ericzon BG. Familial Amyloidotic Polyneuropathy World Transplant Registry. Ten years of international experience with liver transplantation for familial amyloidotic polyneuropathy: results from the Familial Amyloidotic Polyneuropathy World Transplant Registry. Transplantation 77(1), 64-71 (2004).

53 Suhr OB, Larsson M, Ericzon BG, Wilczek HE. FAPWTR's Investigators. Survival after transplantation in patients with mutations other than Val30Met: extracts from the FAP World Transplant Registry. Transplantation 100(2), 373-381 (2016).

54 Drent G, Graveland CW, Hazenberg BP, Haagsma EB. Quality of life in patients with familial amyloidotic polyneuropathy long-term after liver transplantation. Amyloid 16(3), 133-141 (2009).

55 Adams D, Buades J, Suhr O, Obici L, Coelho T. Preliminary assessment of neuropathy progression in patients with hereditary ATTR amyloidosis after orthotopic liver transplantation. Orphanet. J. Rare Dis. 10(Suppl. 1), 25 (2015).

56 Liepnieks JJ, Zhang LQ, Benson MD. Progression of transthyretin amyloid neuropathy after liver transplantation. Neurology 75(4), 324-327 (2010).

57 Hara R, Kawaji T, Ando E, Ohya Y, Ando Y, Tanihara H. Impact of liver transplantation on transthyretin-related ocular amyloidosis in Japanese patients. Arch. Ophthalmol. 128(2), 206-210 (2010).

58 Sekijima Y, Yazaki M, Oguchi K et al. Cerebral amyloid angiopathy in posttransplant patients with hereditary ATTR amyloidosis. Neurology 87(8), 773-781 (2016).

59 Yao FY, Gautam M, Palese C et al. De novo malignancies following liver transplantation: a case-control study with long-term follow-up. Clin. Transplant. 20(5), 617-623 (2006).

60 Said G, Grippon S, Kirkpatrick P. Tafamidis. Nat. Rev. Drug Discov. 11(3), 185-186 (2012).

61 European Medicines Agency. Summary of product characteristics: Vyndaqel 2018. www.ema.europa.eu/docs/en_GB/document_library/EPAR_-_Product_Information/human/002294/WC500117862.pdf

62 Coelho T, Maia LF, Martins da Silva A et al. Tafamidis for transthyretin familial amyloid polyneuropathy: a randomized, controlled trial. Neurology 79(8), 785-792 (2012). 
63 Lozeron P, Theaudin M, Mincheva Z et al. Effect on disability and safety of tafamidis in late onset of Met30 transthyretin familial amyloid polyneuropathy. Eur. J. Neurol. 20(12), 1539-1545 (2013).

64 Ando Y, Sekijima Y, Obayashi K et al. Effects of tafamidis treatment on transthyretin (TTR) stabilization, efficacy, and safety in Japanese patients with familial amyloid polyneuropathy (TTR-FAP) with Val30Met and non-Val30Met: a Phase III, open-label study. J. Neurol. Sci. 362, 266-271 (2016).

65 Cortese A, Vita G, Luigetti M et al. Monitoring effectiveness and safety of tafamidis in transthyretin amyloidosis in Italy: a longitudinal multicenter study in a non-endemic area. J. Neurol. 263(5), 916-924 (2016).

66 Plante-Bordeneuve V, Gorram F, Salhi H et al. Long-term treatment of transthyretin familial amyloid polyneuropathy with tafamidis: a clinical and neurophysiological study. J. Neurol. 264(2), 268-276 (2017).

67 Maurer MS, Schwartz JH, Gundapaneni B et al. Tafamidis treatment for patients with transthyretin amyloid cardiomyopathy. N. Engl. J. Med. 379(11), 1007-1016 (2018).

68 Sekijima Y, Dendle MA, Kelly JW. Orally administered diflunisal stabilizes transthyretin against dissociation required for amyloidogenesis. Amyloid 13(4), 236-249 (2006).

69 Berk JL, Suhr OB, Obici L et al. Repurposing diflunisal for familial amyloid polyneuropathy: a randomized clinical trial. JAMA 310(24), 2658-2667 (2013).

70 Quarta CC, Falk RH, Solomon SD et al. The prevalence of cardiac amyloidosis in familial amyloidotic polyneuropathy with predominant neuropathy: the diflunisal trial. XVth International Symposium on Amyloidosis (ISA), Indianapolis, IN, USA, April 27-May 12014.

71 Castano A, Helmke S, Alvarez J, Delisle S, Maurer MS. Diflunisal for ATTR cardiac amyloidosis. Congest. Heart Fail. 18(6), 315-319 (2012).

72 Sekijima Y, Tojo K, Morita H, Koyama J, Yazaki M, Ikeda S. Safety and efficacy of long-term diflunisal administration in familial amyloid polyneuropathy - summary of ten years therapeutic experience. Presented at: XVth International Symposium on Amyloidosis (ISA), Indianapolis, IN, USA, 27 April-1 May 2014.

73 Hernandez-Diaz S, Rodriguez LA. Association between nonsteroidal anti-inflammatory drugs and upper gastrointestinal tract bleeding/perforation: an overview of epidemiologic studies published in the 1990s. Arch. Intern. Med. 160(14), 2093-2099 (2000)

74 Reddy KS, Roy A. Cardiovascular risk of NSAIDs: time to translate knowledge into practice. PLoS Med. 10(2), e1001389 (2013).

75 Singh BK, Haque SE, Pillai KK. Assessment of nonsteroidal anti-inflammatory drug-induced cardiotoxicity. Expert Opin. Drug Metab. Toxicol. 10(2), 143-156 (2014).

76 European Medicines Agency. Summary of product characteristics: Tegsedi 284mg solution for injection in pre-filled syringe (2018). www.ema.europa.eu/documents/product-information/tegsedi-epar-product-information_en.pdf

77 Akcea Therapeutics Inc. US prescribing information: TEGSEDI ${ }^{\text {TM }}$ (inotersen) injection, for subcutaneous use (2018). https://tegsedi.com/wp-content/uploads/2018/10/prescribing-information.pdf

78 Benson MD, Waddington-Cruz M, Berk JL et al. Inotersen treatment for patients with hereditary transthyretin amyloidosis. N. Engl. J. Med. 379(1), 22-31 (2018).

79 Cardoso I, Martins D, Ribeiro T, Merlini G, Saraiva MJ. Synergy of combined doxycycline/TUDCA treatment in lowering Transthyretin deposition and associated biomarkers: studies in FAP mouse models. J. Transl. Med. 8, 74 (2010).

80 Obici L, Perlini S, Palladini G et al. A Phase II study of doxycycline plus tauroursodeoxycholic acid in transthyretin amyloidosis. XIVth International Symposium on Amyloidosis (ISA), Uppsala, Sweden (2016). Poster PA96.

81 Galant NJ, Bugyei-Twum A, Rakhit R et al. Substoichiometric inhibition of transthyretin misfolding by immune-targeting sparsely populated misfolding intermediates: a potential diagnostic and therapeutic for TTR amyloidoses. Sci. Rep. 6, 25080 (2016).

82 Higaki JN, Chakrabartty A, Galant NJ et al. Novel conformation-specific monoclonal antibodies against amyloidogenic forms of transthyretin. Amyloid 23(2), 86-97 (2016).

83 Holmgren G, Steen L, Ekstedt J et al. Biochemical effect of liver transplantation in two Swedish patients with familial amyloidotic polyneuropathy $\left(\mathrm{FAP}-\mathrm{met}^{30}\right.$ ). Clin. Genet. 40(3), 242-246 (1991).

84 Soprano DR, Herbert J, Soprano KJ, Schon EA, Goodman DS. Demonstration of transthyretin mRNA in the brain and other extrahepatic tissues in the rat. J. Biol. Chem. 260(21), 11793-11798 (1985).

85 Alnylam Pharmaceuticals. US prescribing information: ONPATTRO (patisiran) lipid complex injection, for intravenous use. www.accessdata.fda.gov/drugsatfda_docs/label/2018/210922s000lbl.pdf

86 European Medicines Agency. Summary of opinion (initial authorisation): Onpattro (patisiran) (2018). www.ema.europa.eu/documents/smop-initial/chmp-summary-positive-opinion-onpattro_en.pdf

87 Haagsma EB, Van Gameren I, Bijzet J, Posthumus MD, Hazenberg BP. Familial amyloidotic polyneuropathy: long-term follow-up of abdominal fat tissue aspirate in patients with and without liver transplantation. Amyloid 14(3), 221-226 (2007).

88 Tsuchiya A, Yazaki M, Kametani F, Takei Y, Ikeda S. Marked regression of abdominal fat amyloid in patients with familial amyloid polyneuropathy during long-term follow-up after liver transplantation. Liver Transpl. 14(4), 563-570 (2008). 
89 Lachmann HJ, Gallimore R, Gillmore JD et al. Outcome in systemic AL amyloidosis in relation to changes in concentration of circulating free immunoglobulin light chains following chemotherapy. Br. J. Haematol. 122(1), 78-84 (2003).

90 Palladini G, Dispenzieri A, Gertz MA et al. New criteria for response to treatment in immunoglobulin light chain amyloidosis based on free light chain measurement and cardiac biomarkers: impact on survival outcomes. J. Clin. Oncol. 30(36), 4541-4549 (2012).

91 Lachmann HJ, Goodman HJ, Gilbertson JA et al. Natural history and outcome in systemic AA amyloidosis. N. Engl. J. Med. 356(23), 2361-2371 (2007).

92 Gillmore JD, Lovat LB, Persey MR, Pepys MB, Hawkins PN. Amyloid load and clinical outcome in AA amyloidosis in relation to circulating concentration of serum amyloid A protein. Lancet 358(9275), 24-29 (2001).

93 Adams D, Samuel D, Goulon-Goeau C et al. The course and prognostic factors of familial amyloid polyneuropathy after liver transplantation. Brain 123(Pt 7), 1495-1504 (2000).

94 Hannon GJ. RNA interference. Nature 418(6894), 244-251 (2002).

95 Coelho T, Adams D, Silva A et al. Safety and efficacy of RNAi therapy for transthyretin amyloidosis. N. Engl. J. Med. 369(9), 819-829 (2013).

-. Report of the proof of principle for patisiran in human subjects from a Phase I dose-ascending study.

96 Crooke ST. Antisense strategies. Curr. Mol. Med. 4(5), 465-487 (2004).

97 Elbashir SM, Harborth J, Lendeckel W, Yalcin A, Weber K, Tuschl T. Duplexes of 21-nucleotide RNAs mediate RNA interference in cultured mammalian cells. Nature 411(6836), 494-498 (2001).

98 Huang SK, Mayhew E, Gilani S, Lasic DD, Martin FJ, Papahadjopoulos D. Pharmacokinetics and therapeutics of sterically stabilized liposomes in mice bearing C-26 colon carcinoma. Cancer Res. 52(24), 6774-6781 (1992).

99 Kulkarni JA, Cullis PR, van der Meel R. Lipid nanoparticles enabling gene therapies: from concepts to clinical utility. Nucleic Acid Ther. 28(3), 146-157 (2018).

100 Goncalves P, Martins H, Costelha S, Saraiva MJ. Efficiency of siRNA for removal of transthyretin V30M in a TTR leptomeningeal animal model. Amyloid 24(Suppl. 1), 38-39 (2017).

101 Suhr OB, Coelho T, Buades J et al. Efficacy and safety of patisiran for familial amyloidotic polyneuropathy: a Phase II multi-dose study. Orphanet. J. Rare Dis. 10, 109 (2015).

-• Pharmacodynamic, pharmacokinetic and safety data of patisiran in a dose-escalation Phase II study in patients with hATTR amyloidosis.

102 Adams D, Gonzalez-Duarte A, O’Riordan WD et al. Patisiran, an RNAi therapeutic, for hereditary transthyretin amyloidosis. N. Engl. J. Med. 379(1), 11-21 (2018).

-. Pivotal Phase III study (APOLLO) of patisiran, demonstrating efficacy and safety of continuous patisiran treatment in patients with hATTR amyloidosis.

103 Berk J, Adams D, Suhr O et al. Long-term, open-label clinical experience with patisiran, an investigational RNAi therapeutic for patients with hereditary transthyretin-mediated (hATTR) amyloidosis with polyneuropathy. Orphanet. J. Rare Dis. 12(Suppl. 1), 165 (2017).

104 Coelho T, Suhr OB, Conceicao I et al. Relationship between TTR knockdown and change in mNIS+7: Preliminary correlation findings from the phase 2 open-label extension study of patisiran, an investigational RNAi therapeutic for hereditary ATTR amyloidosis with polyneuropathy. Presented at: XIVth International Symposium On Amyloidosis (ISA), Uppsula, Sweden, 3-7 July 2016.

105 Adams D, Coelho T, Conceicao I et al. Phase 2 open-label extension (OLE) study of patisiran, an investigational RNAi therapeutic for the treatment of polyneuropathy due to hereditary ATTR (hATTR) amyloidosis: Final 24-month data. American Academy of Neurology (AAN), Boston, MA, USA April 22-28 2017.

106 Adams D, Suhr OB, Dyck PJ et al. Trial design and rationale for APOLLO, a Phase 3, placebo-controlled study of patisiran in patients with hereditary ATTR amyloidosis with polyneuropathy. BMC. Neurol. 17(1), 181 (2017).

107 Vinik EJ, Vinik AI, Paulson JF et al. Norfolk QOL-DN: validation of a patient reported outcome measure in transthyretin familial amyloid polyneuropathy. J. Peripher. Nerv. Syst. 19(2), 104-114 (2014).

108 Solomon SD, Adams D, Kristen A et al. Effects of patisiran, an RNA interference therapeutic, on cardiac parameters in patients with hereditary transthyretin-mediated amyloidosis: an analysis of the APOLLO study. Circulation (2018) (In press).

109 Quarta CC, Solomon SD, Uraizee I et al. Left ventricular structure and function in transthyretin-related versus light-chain cardiac amyloidosis. Circulation 129(18), 1840-1849 (2014).

110 Suhr OB, Gonzalez-Duarte A, O'Riordan W et al. Long-term use of patisiran, an investigational RNAi therapeutic, in patients with hereditary transthyretin-mediated amyloidosis: baseline demographics and interim data form global open label extension study. Presented at: XVIth International Symposium On Amyloidosis (ISA), Kumamoto, Japan, 26-29 March 2018.

111 Adams D, Gonzalez-Duarte A, O’Riordan W et al. Patisiran, an investigational RNAi therapeutic for the treatment of hereditary ATTR amyloidosis with polyneuropathy: results from the Phase 3 APOLLO study. Orphanet. J. Rare Dis. 12(Suppl. 1), 165 (2017).

112 Polydefkis M, Adams D, Kristen A et al. Infusion Related Reactions In Patients With hATTR Amyloidosis Treated With Patisiran. Peripheral Nerve Society (PNS), Baltimore, MD, USA (2018). 
113 Taubel J, Zimmermann T, Karsten V et al. Phase 1 study of ALN-TTRsc02, a subcutaneously administered investigational RNAi therapeutic for the treatment of transthyretin-mediated amyloidosis. Presented at: XVIth International Symposium On Amyloidosis (ISA). Kumamoto, Japan, 26-29 March 2018. 
